\title{
Effects of nonadiabaticity on the voltage generated by a moving domain wall
}

\author{
R. A. Duine* \\ Institute for Theoretical Physics, Utrecht University, Leuvenlaan 4, 3584 CE Utrecht, The Netherlands \\ (Received 19 September 2008; revised manuscript received 2 December 2008; published 7 January 2009)
}

\begin{abstract}
We determine the voltage generated by a field-driven transverse domain wall, taking into account nonadiabatic corrections to the motive force induced by the time-dependent spin Berry phase. Both the diffusive and ballistic transport regimes are considered. We find that the nonadiabatic corrections, together with the contributions due to spin relaxation, determine the voltage for driving fields smaller than the Walker breakdown limit.
\end{abstract}

DOI: 10.1103/PhysRevB.79.014407

PACS number(s): 72.25.Pn, 72.15.Gd

\section{INTRODUCTION AND SUMMARY OF RESULTS}

This paper develops the theory of nonadiabatic corrections to the voltage generated by a moving domain wall. This aim is primarily motivated by the fact that in certain situations, for example, that of a narrow wall, these corrections are important for the correct description of current-driven domain-wall motion. We find that nonadiabatic corrections play an important role in the reverse process as well.

This introductory section is intended to be self-contained. The more technical sections can be consulted for details of the various calculations. We discuss current-driven magnetization dynamics and the reverse process-generation of current by a time-dependent magnetization texture-in two separate subsections. Our main results are presented and discussed at the end of this section.

\section{A. Current-driven magnetization dynamics}

Let us consider a ferromagnetic metallic wire far below the Curie temperature, characterized by a unit vector $\mathbf{\Omega}(\mathbf{x}, t)$ in the direction of magnetization. Suppose we drive an electric current through the wire, say, in the $x$ direction and the magnetization direction only varies in this direction. In the adiabatic limit, that is, when the inverse Fermi wave number $k_{F}$ or the electron mean free path is much smaller than the typical length scale of magnetization-direction variation, the electric transport current is spin polarized in the local direction of magnetization. As the electrons traverse a noncollinear part of the ferromagnet, where the magnetization direction changes from $\boldsymbol{\Omega}(x, t)$ to $\boldsymbol{\Omega}(x+d x, t)$, the conduction electrons experience a torque that changes their spinpolarization direction. This torque is exerted by the magnetization. Conversely, there is a reaction torque on the magnetization given by

$$
\left.\frac{\partial \boldsymbol{\Omega}(x, t)}{\partial t}\right|_{\text {current }} \propto \boldsymbol{\Omega}(x+d x, t)-\boldsymbol{\Omega}(x, t) \propto \frac{\partial \boldsymbol{\Omega}(x, t)}{\partial x}
$$

called a spin-transfer torque. ${ }^{1-7}$ This expression hinges on conservation of total spin. Roughly speaking, the magnetization and conduction-electron-spin precess around each other while conserving their total spin angular momentum. When there is electron-spin relaxation, in metals primarily due to spin-orbit coupling and spin-flip scattering events, there is an additional current-induced torque. In the adiabatic limit this torque turns out to be in the form of $\boldsymbol{\Omega}(x, t) \times \partial \boldsymbol{\Omega} / \partial x$, which is understood from the requirement that it should be perpendicular both to the magnetization direction and to the torque in Eq. (1). In the adiabatic limit the torques due to the current are sum of these two contributions and are given by

$$
\begin{aligned}
\left.\frac{\partial \boldsymbol{\Omega}(x, t)}{\partial t}\right|_{\text {current }}= & \left(\mathbf{v}_{s} \cdot \boldsymbol{\nabla}\right) \boldsymbol{\Omega}(\mathbf{x}, t)+\beta_{\mathrm{sr}} \mathbf{\Omega}(\mathbf{x}, t) \\
& \times\left(\mathbf{v}_{s} \cdot \boldsymbol{\nabla}\right) \boldsymbol{\Omega}(\mathbf{x}, t),
\end{aligned}
$$

with $\beta_{\mathrm{sr}}$ as a dimensionless coefficient that characterizes the degree to which spin is not conserved in the spin-transfer process. ${ }^{8-13}$ The velocity $\mathbf{v}_{s}=-\mathcal{P} \mathbf{j} /|e| \rho_{s}$ is proportional to the current $\mathbf{j}$ (with $\mathcal{P}$ as the current spin polarization, $\rho_{s}$ as the density difference of majority and minority-spin electrons, and $-|e|$ as the electron charge). The two terms on the righthand side of the above equation are properly called the reactive and dissipative adiabatic spin-transfer torques, ${ }^{13-15}$ respectively, although they are also referred to as adiabatic and nonadiabatic for reasons that will become clear shortly.

In a series of papers, Tatara et al. ${ }^{16-18}$ also considered nonadiabatic corrections to Eq. (2), ${ }^{19}$ which quite generally contribute a nonlocal term to Eq. (2) so that it becomes

$$
\begin{aligned}
\left.\frac{\partial \boldsymbol{\Omega}(x, t)}{\partial t}\right|_{\text {current }} & \left(\mathbf{v}_{s} \cdot \boldsymbol{\nabla}\right) \boldsymbol{\Omega}(\mathbf{x}, t)+\beta_{\mathrm{sr}} \boldsymbol{\Omega}(\mathbf{x}, t) \\
& \times\left(\mathbf{v}_{s} \cdot \boldsymbol{\nabla}\right) \boldsymbol{\Omega}(\mathbf{x}, t)+\Gamma_{\mathrm{n} a}[\mathbf{\Omega}],
\end{aligned}
$$

with $\Gamma_{\text {na }}[\boldsymbol{\Omega}]$ as a functional that can in principle be evaluated in certain limits. (See also Refs. 20-22 for a treatment of nonadiabatic corrections.) In particular, Tatara et al. ${ }^{16-18}$ considered the effect of momentum transfer, which corresponds physically to electrons scattering off the magnetization texture. The evaluation of the nonlocal torque $\Gamma[\mathbf{\Omega}]$ that corresponds to this process is quite complicated for a general magnetization texture. Motivated by ongoing experimental $^{23-30}$ and theoretical ${ }^{8-12,16-18,20,22,31-35}$ researches on current-driven motion of domain walls, Tatara et $a l .{ }^{16-18}$ evaluated Eq. (3) within a simple variational description of the domain wall. Namely, they showed that for a straight rigid domain wall Eq. (3) yields

$$
\left.\frac{d \phi_{0}(t)}{d t}\right|_{\text {current }}=\left(\beta_{\mathrm{sr}}+\beta_{\mathrm{na}}\right) \frac{v_{s}}{\lambda},
$$




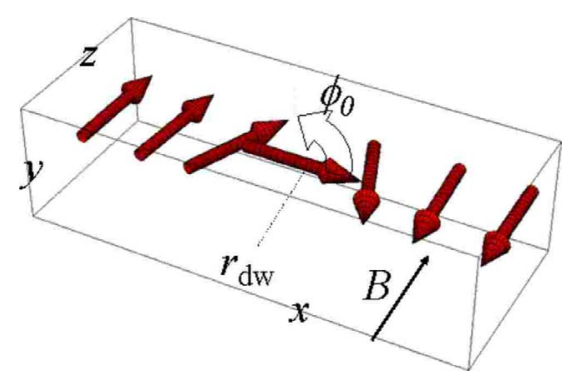

FIG. 1. (Color online) Illustration of a domain wall at position $r_{\mathrm{dw}}(t)$ in a magnetic field $B$ pointing in the $z$ direction. The chirality $\phi_{0}(t)$ is the angle of the spin at the position of the domain wall with the $x-y$ plane.

$$
\left.\frac{d r_{\mathrm{dw}}(t)}{d t}\right|_{\text {current }}=v_{s}
$$

where the current is taken in the direction of the domain wall, i.e., in the direction of varying magnetization. In the above expression the dynamical variational parameters $r_{\mathrm{dw}}(t)$ and $\phi_{0}(t)$ denote the position of the domain wall and its chirality, respectively. For the case of an easy-plane ferromagnet, for example, $\phi_{0}(t)$ is the canting angle with which the magnetization tilts out of the easy plane at the domainwall position. Furthermore, $\lambda$ is the width of the domain wall, determined by the competition between exchange and anisotropy. See Fig. 1 for an illustration.

In the clean limit, the coefficient $\beta_{\mathrm{na}}$ is found as ${ }^{17}$

$$
\beta_{\mathrm{na}}=\frac{\lambda}{\mathcal{P} A} \frac{|e|^{2}}{\hbar} N_{e} \rho_{\mathrm{dw}},
$$

with $A$ is the cross section of the magnetic wire perpendicular to the domain wall and current direction, $N_{e}$ is the number of electrons, and $\rho_{\mathrm{dw}}$ is the contribution to the resistivity due to the domain wall.

Interestingly, the momentum-transfer process, described by a complicated nonlocal term in Eq. (3) for the full magnetization-direction dynamics, yields a simple renormalization of $\beta_{\mathrm{sr}}$ at the level of the variational description in Eq. (4). ${ }^{18}$ [This is the reason for referring to the term proportional to $\beta_{\text {sr }}$ in Eq. (3) as "nonadiabatic," which is strictly speaking incorrectly.] The main result of this paper is that the same renormalization occurs for the case of charge-current generation by a moving domain wall.

We end this subsection by mentioning that, over the last few years, Eqs. (3) and (4) have been actively debated. In particular, the ratio of the coefficient $\beta_{\text {sr }}$ to the so-called Gilbert damping constant $\alpha_{G}$ that governs magnetization relaxation has been a subject of interest. Although it is now generally accepted that this ratio is generally not equal to 1 , as indicated by microscopic theories ${ }^{11,13}$ and recent experiments, ${ }^{36}$ characterizing and optimizing the various processes that lead to current-driven domain-wall motion will most likely remain an active topic of research in the near future.

\section{B. Current generated by a time-dependent magnetization texture}

The reverse process of current-driven magnetization dynamics is the generation of current and voltage by a timedependent magnetization texture. The expression for the charge current is given by ${ }^{37-40}$

$$
\begin{aligned}
j_{\alpha}= & -\frac{\hbar}{2|e| V}\left(\sigma_{\uparrow}-\sigma_{\downarrow}\right)\left\{\frac{\partial}{\partial t} \int d \mathbf{x} \tilde{A}_{\alpha^{\prime}}[\mathbf{\Omega}(\mathbf{x}, t)] \nabla_{\alpha} \Omega_{\alpha^{\prime}}(\mathbf{x}, t)\right. \\
& \left.+\beta_{\mathrm{sr}} \int d \mathbf{x} \frac{\partial \mathbf{\Omega}(\mathbf{x}, t)}{\partial t} \cdot \nabla_{\alpha} \mathbf{\Omega}(\mathbf{x}, t)+\mathcal{E}_{\mathrm{na}}(\mathbf{\Omega})\right\},
\end{aligned}
$$

with $V$ as the volume of the system and $\sigma_{\uparrow}$ and $\sigma_{\downarrow}$ as the respective conductivities of the majority and minority-spin electrons. In this paper, a summation over repeated indices $\alpha, \alpha^{\prime}, \alpha^{\prime \prime} \in\{x, y, z\}$ is implied.

The three terms in the above equation correspond, roughly speaking, to the three terms on the right-hand side of Eq. (3), respectively. That is, the first term is adiabatic and, loosely speaking, is due to conservation of spin. It is given in terms of a vector potential $\widetilde{A}_{\alpha}(\boldsymbol{\Omega})$ of a magnetic monopole in spin space (not to be confused with the electromagnetic vector potential $\mathbf{A}$ that we will introduce later on) that obeys $\epsilon_{\alpha, \alpha^{\prime}, \alpha^{\prime \prime}} \partial \widetilde{A}_{\alpha^{\prime}} / \partial \Omega_{\alpha^{\prime \prime}}=\Omega_{\alpha}$ and is well known from the pathintegral formulation for spin systems. ${ }^{41}$ This term corresponds to the time derivative of the flux of a monopole magnetic field (in spin space) enclosed by the path $\boldsymbol{\Omega}(\mathbf{x}, t)$ on the unit sphere and is the motive force induced by the timedependent spin Berry phase. It was first discussed by Stern ${ }^{37}$ and later by Barnes and Maekawa ${ }^{38}$ in the context of Faraday's law in a ferromagnetic metal.

Before discussing the remaining two contributions to the generated current, we give, for completeness and future reference, first an expression for the voltage drop $\Delta V$ in the $\alpha$ direction across a wire of length $L$ with cross-sectional area $A$. Using $\Delta V=j_{\alpha} L /\left(\sigma_{\uparrow}+\sigma_{\downarrow}\right)$, we find

$$
\begin{aligned}
\Delta V= & -\frac{\hbar \mathcal{P}}{2|e| A}\left\{\int d \mathbf{x} \boldsymbol{\Omega}(\mathbf{x}, t) \cdot\left[\frac{\partial \mathbf{\Omega}(\mathbf{x}, t)}{\partial t} \times \nabla_{\alpha} \boldsymbol{\Omega}(\mathbf{x}, t)\right]\right. \\
& \left.+\beta_{\mathrm{sr}} \int d \mathbf{x} \frac{\partial \mathbf{\Omega}(\mathbf{x}, t)}{\partial t} \cdot \nabla_{\alpha} \boldsymbol{\Omega}(\mathbf{x}, t)+\mathcal{E}_{\mathrm{na}}[\mathbf{\Omega}]\right\}
\end{aligned}
$$

where we used the properties of the vector potential $\tilde{\mathbf{A}}(\boldsymbol{\Omega})$ to work out the first term in Eq. (6) and $\mathcal{P} \equiv\left(\sigma_{\uparrow}-\sigma_{\downarrow}\right) /\left(\sigma_{\uparrow}\right.$ $\left.+\sigma_{\downarrow}\right)$. Note that in deriving the expression for the voltage we have assumed that the total conductivity is given by $\sigma_{\uparrow}+\sigma_{\downarrow}$ and have therefore neglected other contributions, e.g., due to the presence of a domain wall and/or anisotropic magnetoresistance. We will come back to this point in Secs. II-IV.

The second term in Eqs. (6) and (7) was first derived in Ref. 39 using response-function techniques. It corresponds to an adiabatic correction, due to spin-orbit coupling and spinflip scattering, to the Berry-phase-induced motive force (which is also adiabatic). The same correction was found by Tserkovnyak and Mecklenburg ${ }^{40}$ using Onsager reciprocity. We mention also the work by Saslow ${ }^{42}$ who considered the generation of electric current by time-dependent magnetiza- 
tion textures in the absence of conservation of spin within the framework of irreversible thermodynamics.

The last contribution to Eqs. (6) and (7), proportional to $\mathcal{E}_{\text {na }}[\boldsymbol{\Omega}]$, corresponds formally to all terms beyond linear order in spatial gradients of the magnetization direction. Although, to the best of our knowledge, it has not been considered in great detail, it could be calculated, for example, order by order in a gradient expansion. The main result of this paper is that, when Eqs. (6) and (7) are evaluated within the same description that yields Eq. (4) from Eq. (3), one finds that $^{43}$

$$
\Delta V=-\frac{\hbar}{|e|}\left(\frac{\sigma_{\uparrow}-\sigma_{\downarrow}}{\sigma_{\uparrow}+\sigma_{\downarrow}}\right)\left[\dot{\phi}_{0}(t)-\frac{\left(\beta_{\mathrm{sr}}+\beta_{\mathrm{na}}\right) \dot{r}_{\mathrm{dw}}(t)}{\lambda}\right] .
$$

Hence, taking into account nonadiabatic corrections to the generated current again amounts to the renormalization $\beta_{\mathrm{sr}}$ $\rightarrow \beta_{\mathrm{sr}}+\beta_{\mathrm{na}}$ at the level of the effective (variational) model. To arrive at this result it turns out to be sufficient to assume Eq. (4) from which the above result can be straightforwardly derived, as we show in the Sec. II.

Next, we give the result for the evaluation of Eq. (8) for the case of a field-driven domain wall with an easy plane and a hard axis. In this case, the wall moves with constant velocity below the so-called Walker breakdown field $B_{W}{ }^{44}$ This field is proportional to the hard axis anisotropy energy and to the Gilbert damping constant $\alpha_{G}$. The domain-wall precession angle is time independent for fields $B$ smaller than $B_{W}$. Above this field the domain-wall chirality becomes time dependent and the domain wall undergoes oscillatory motion. The result in Eq. (8) leads to ${ }^{39}$

$$
\begin{aligned}
\frac{\Delta V}{V_{0}}= & \frac{\left(\beta_{\mathrm{sr}}+\beta_{\mathrm{na}}\right)}{\alpha_{G}} \frac{B}{B_{W}} \\
& -\left(\frac{1+\frac{\left(\beta_{\mathrm{sr}}+\beta_{\mathrm{na}}\right)}{\alpha_{G}}}{1+\alpha_{G}^{2}}\right) \operatorname{Re}\left[\sqrt{\left(\frac{B}{B_{W}}\right)^{2}-1}\right],
\end{aligned}
$$

with $V_{0}=\mathcal{P} g \mu_{B} B_{W} /|e|$, in terms of the gyromagnetic ratio $g$ and the Bohr magneton $\mu_{B}$. For typical experiments ${ }^{45}\left(B_{W}\right.$ $\sim 100$ Oe) we have that $V_{0} \sim 0.5 \mu \mathrm{V}$. Note that $V_{0}$ is roughly the Walker breakdown field converted to a voltage. In Fig. 2 we plot this result for various values of $\left(\beta_{\mathrm{sr}}\right.$ $\left.+\beta_{\text {na }}\right) / \alpha_{G}$. From this figure, and also from the expression in Eq. (9), it is obvious that below Walker breakdown the induced voltage is completely determined by the spin-orbit coupling and spin-flip scattering and by the nonadiabatic corrections.

In Sec. II we derive our main result in Eq. (8). In Sec. III we investigate how the two contributions to the generated voltage, proportional to $d r_{\mathrm{dw}} / d t$ and $d \phi_{0} / d t$, arise in a ballistic model for electron transport. We end in Sec. IV with a short discussion and outlook. Before we turn to the more technical part of this paper, let us end this section with a brief description of other work on the generation of charge current by a time-dependent magnetization. Already, in 1986, Berger $^{46}$ discussed the generation of current by moving domain walls in terms of an analog of the Josephson effect. The motive force due to the time-dependent spin Berry phase was

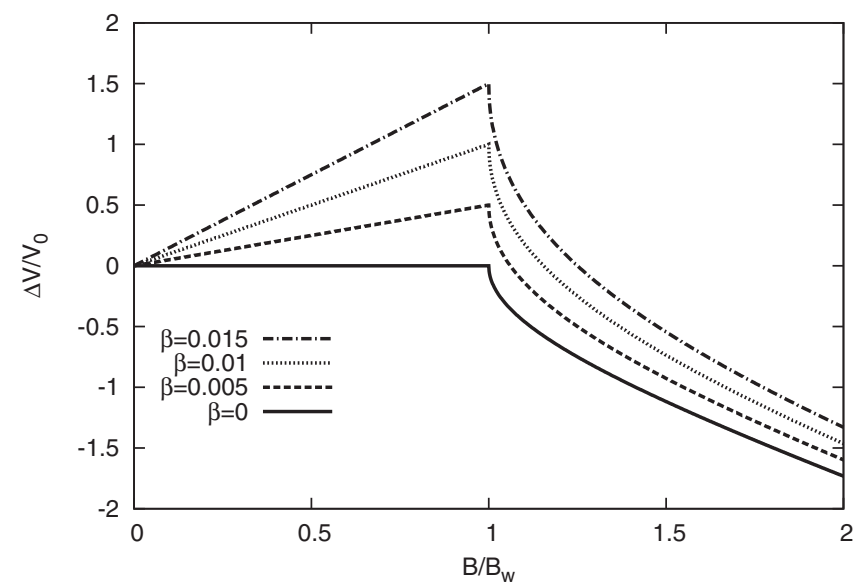

FIG. 2. Voltage induced by a moving domain wall for Gilbert damping $\alpha_{G}=0.01$ and various values of $\beta \equiv \beta_{\mathrm{sr}}+\beta_{\text {na }}$. The voltage is normalized to $V_{0}=\mathcal{P} g \mu_{B} B_{W} /|e|$. The magnetic field $B$ is in unit of the Walker breakdown field $B_{W}$. For typical experiments we have that $V_{0} \sim 0.5 \mu \mathrm{V}$.

first pointed out by $\mathrm{Stern}^{37}$ in the context of a mesoscopic ring with a Zeeman magnetic field, and later considered in the context of domain walls by Barnes and Maekawa. ${ }^{38}$ Both these works did not consider the effects of spin-orbit coupling and spin-flip scattering in the adiabatic limit. This was done first in Ref. 39, by Saslow, ${ }^{42}$ and by Tserkovnyak and Mecklenburg. ${ }^{40}$ The limit of strong Rashba spin-orbit coupling was considered by Ohe et al. ${ }^{47}$ Yang et al. ${ }^{48}$ evaluated Eq. (7) with $\beta_{\mathrm{sr}}=0$ and without nonadiabatic corrections using results of micromagnetic simulations for straight and vortex domain walls. Stamenova et al. ${ }^{49}$ performed a detailed numerical analysis of the Berry-phase-induced motive force, also without considering spin-orbit coupling or spin-flip scattering.

Very recently, the electromotive force has been measured for vortex-type domain walls ${ }^{50}$ in which the nonadiabatic effects, which are the main focus of the present paper, likely play a less important role than in straight domain walls. Following this very important experimental result, we expect that more experimental results will be reported on in the near future.

\section{DIFFUSIVE TRANSPORT REGIME}

In the diffusive transport regime, we assume that the ferromagnetic metal is characterized by conductivities $\sigma_{\uparrow}$ and $\sigma_{\downarrow}$ for the majority and minority-spin bands, respectively. Furthermore, we assume that the velocity $v_{s}$ is given by the linear-response expression

$$
v_{s}=-\frac{\left(\sigma_{\uparrow}-\sigma_{\downarrow}\right) E}{|e| \rho_{s}},
$$

where the electric field $E$ is taken in the domain-wall direction. The easiest way to proceed is by writing down an action $\mathcal{A}\left[r_{\mathrm{dw}}, \phi_{0}\right]$ that, upon variation, reproduces the equation of motion for the variational parameters in Eq. (4). Using the short-hand notation 


$$
\beta \equiv\left(\beta_{\mathrm{sr}}+\beta_{\mathrm{na}}\right),
$$

we have in imaginary time $\tau=i t$ that

$$
\begin{aligned}
\mathcal{A}\left[r_{\mathrm{dw}}, \phi_{0}\right]= & \int_{0}^{\hbar / k_{B} T} d \tau N\left[i \hbar \frac{r_{\mathrm{dw}}(\tau)}{\lambda} \frac{d \phi_{0}(\tau)}{d \tau}+\hbar \frac{v_{s}}{\lambda} \phi_{0}(\tau)\right. \\
& \left.-\hbar \beta v_{s} \frac{r_{\mathrm{dw}}(\tau)}{\lambda^{2}}\right],
\end{aligned}
$$

with $k_{B} T$ as the thermal energy. Here, $N=\lambda A \rho_{s}$ is the number of electron spins in the domain wall. In principle, the action for the domain wall contains potential-energy terms due to anisotropy, inhomogeneities, and external magnetic field. They are, however, not important in describing the coupling of the current to the domain wall and are omitted in the above expression [as well as in Eq. (4)].

Although the above action can in principle be derived microscopically within a given approximation scheme, this is not needed here. All we need to extract the contributions to the current due to the moving domain wall is the fact that there exists a response function $\Pi\left(\mathbf{x}, \mathbf{x}^{\prime} ; \tau-\tau^{\prime}\right)$ such that

$$
v_{s}=\int_{0}^{\hbar / k_{B} T} d \tau^{\prime} \int d \mathbf{x} \int d \mathbf{x}^{\prime} \Pi\left(\mathbf{x}, \mathbf{x}^{\prime} ; \tau-\tau^{\prime}\right) A\left(\mathbf{x}^{\prime}, \tau^{\prime}\right)
$$

reduces to Eq. (10) for a vector potential,

$$
A(\mathbf{x}, \tau)=-\frac{c E}{\omega_{p}} e^{-i \omega_{p} \tau},
$$

when we take the zero-frequency limit $\omega_{p} \rightarrow 0$. Here, $c$ is the velocity of light. This requirement determines the lowfrequency behavior of the response function $\widetilde{\Pi}\left(i \omega_{n}\right)$ defined by

$$
\frac{1}{\hbar / k_{B} T} \sum_{n} \widetilde{\Pi}\left(i \omega_{n}\right) e^{-i \omega_{n}\left(\tau-\tau^{\prime}\right)}=\int d \mathbf{x} \int d \mathbf{x}^{\prime} \Pi\left(\mathbf{x}, \mathbf{x}^{\prime} ; \tau-\tau^{\prime}\right)
$$

as

$$
\widetilde{\Pi}\left(i \omega_{n}\right)=\frac{\left(\sigma_{\uparrow}-\sigma_{\downarrow}\right) \omega_{n}}{c|e| \rho_{s}},
$$

with $\omega_{n}=2 \pi n k_{B} T / \hbar$ as the bosonic Matsubara frequencies. At this point we note that the nonadiabaticity, although somewhat hidden in the formalism, is incorporated by allowing the response function $\Pi\left(\mathbf{x}, \mathbf{x}^{\prime} ; \tau-\tau^{\prime}\right)$ to depend arbitrarily on the spatial coordinates $\mathbf{x}, \mathbf{x}^{\prime}$.

The next ingredient we need is that quite generally the expectation value of the electric current $j$ is given by a functional derivative of the effective action via

$$
j=c \frac{\delta \mathcal{A}\left[r_{\mathrm{dw}}, \phi_{0}\right]}{\delta A(\mathbf{x}, \tau)} .
$$

This yields in first instance for the current

$$
\begin{aligned}
j= & \frac{c}{L} \int_{0}^{\hbar / k_{B} T} d \tau^{\prime} \int d \mathbf{x} \int d \mathbf{x}^{\prime} \rho_{s} \hbar \\
& \times\left\{\left[\phi_{0}\left(\tau^{\prime}\right)-\beta \frac{r_{\mathrm{dw}}\left(\tau^{\prime}\right)}{\lambda}\right] \Pi\left(\mathbf{x}, \mathbf{x}^{\prime} ; \tau^{\prime}-\tau\right)\right\} .
\end{aligned}
$$

Using now the result in Eq. (16) we find in the lowfrequency limit that

$$
j=-i \frac{\hbar}{|e| L}\left(\sigma_{\uparrow}-\sigma_{\downarrow}\right)\left[\frac{d \phi(\tau)}{d \tau}-\beta \frac{d r_{\mathrm{dw}}(\tau)}{\lambda d \tau}\right],
$$

which, after a Wick rotation $\tau=i t$ and realizing that $\Delta V$ $=j L /\left(\sigma_{\uparrow}+\sigma_{\downarrow}\right)$, leads to Eq. (8).

This result is rather general in the sense that it does not depend on specific values of $\alpha_{G}$ and $\beta_{\mathrm{sr}}+\beta_{\text {na }}$ or the microscopic mechanisms contributing to these coefficients. The only input is that Eq. (4), together with Ohm's law in Eq. (10), holds. Moreover, the result is applicable within both the $s-d$ and the Stoner model for ferromagnetism. We emphasize, however, that the result derived in this section does not present a microscopic derivation of an expression for $\beta$. Rather, it relies on the validity of Eq. (4). Finally, it is important to note that in calculating the voltage $\Delta V$, we have neglected the contribution to the conductivity due to the presence of the domain wall. In the ballistic limit, to be discussed in Sec. III, this contribution is accounted for rather straightforwardly.

\section{BALLISTIC LIMIT}

In this section we show how the two contributions to the voltage in Eq. (8), proportional to $d \phi_{0} / d t$ and $d r_{\mathrm{dw}} / d t$, respectively, arise in the ballistic limit where the scattering theory of electronic transport ${ }^{51}$ is applicable. This is instructive as the discussion of the generation of spin and charge currents in mesoscopic systems, called spin pumping and charge pumping, respectively, is usually done within this framework. Applications involve quantum dots ${ }^{52-55}$ and single-domain ferromagnets. ${ }^{56-60}$

The starting point is the expression due to Büttiker et $a .^{57,61}$ who gave the current in terms of derivatives of the scattering matrix

$$
I_{\nu}=-\frac{|e|}{4 \pi i} \sum_{\gamma, \nu^{\prime}} \operatorname{Tr}\left[\mathbf{s}_{\nu \nu^{\prime}}^{\dagger} \cdot \frac{\partial \mathbf{s}_{\nu \nu^{\prime}}}{\partial X_{\gamma}}-\frac{\partial \mathbf{s}_{\nu \nu^{\prime}}^{\dagger}}{\partial X_{\gamma}} \cdot \mathbf{s}_{\nu \nu^{\prime}}\right] \frac{d X_{\gamma}}{d t}
$$

to lowest order in the time derivatives. We note at this point that, although the above expression is first order in time derivatives, it contains all nonadiabatic corrections ${ }^{19}$ because it depends on the scattering matrix which in turn depends on the full magnetization texture $\boldsymbol{\Omega}(x)$ and not only its firstorder spatial derivative $\partial \boldsymbol{\Omega}(x) / \partial x$. (Note, however, that in this section we ignore the contribution due to spin relaxation to the induced current and voltage.) In this expression, $I_{\nu}$ is the current ${ }^{62}$ into reservoir $\nu$, and the sum $\nu^{\prime} \in\{1,2\}$ runs over the left and right reservoirs (lead), denoted by 1 and 2 , respectively. The index $\gamma$ labels the parameters $X_{\gamma}$ that vary in time and change the scattering matrix, and the sum over $\gamma$ is over all such parameters. 
The scattering matrix

$$
\mathbf{S}=\left(\begin{array}{ll}
\mathbf{r}_{11} & \mathbf{t}_{12} \\
\mathbf{t}_{21} & \mathbf{r}_{22}
\end{array}\right),
$$

is given in terms of the reflection amplitudes $\mathbf{r}_{\nu \nu}$ and transmission amplitudes $\mathbf{t}_{\nu \nu^{\prime}}$ that describe transmission from reservoir $\nu^{\prime}$ to $\nu$. These quantities have matrix structure in the space of conduction channels of the leads. In Eq. (20) they are to be evaluated at the Fermi energy $\epsilon_{F}$ of the leads.

Consider now specifically the domain-wall configuration shown in Fig. 1, with $\phi_{0}=0$ and with the domain wall located at the origin. We parametrize this domain-wall magnetization-direction texture by $\boldsymbol{\Omega}_{\mathrm{dw}}(x)$ $=\left[\sin \theta_{\mathrm{dw}}(x), 0, \cos \theta_{\mathrm{dw}}(x)\right]$. Within the simplest two-band mean-field model for the ferromagnetism [which could be extended to more complicated and realistic situations (see Ref. 63)], the wave function $\psi(x) \equiv\left[\psi_{\uparrow}(x), \psi_{\downarrow}(x)\right]$ of the electrons with energy $\epsilon$ moving in the presence of this texture obeys the time-independent Schrödinger equation

$$
\left[-\frac{\hbar^{2}}{2 m} \frac{d^{2}}{d x^{2}}-\frac{\Delta}{2} \boldsymbol{\Omega}_{\mathrm{dw}}(x) \cdot \boldsymbol{\tau}\right] \psi(x)=\epsilon \psi(x),
$$

where $\tau$ is the vector of Pauli matrices and $\Delta$ is the exchange splitting. For simplicity we have taken the system to be one dimensional. We will further assume that the magnetization texture is symmetric (in an obvious sense) around the domain-wall position.

Asymptotically, the scattering state for an electron coming in from the left reservoir with spin state $|\sigma\rangle$ is given by

$$
\psi^{1 \sigma}(x)=\sum_{\sigma^{\prime}}\left[\delta_{\sigma \sigma^{\prime}} e^{i k_{\sigma^{\prime}} x}+\sqrt{\frac{k_{\sigma}}{k_{\sigma^{\prime}}}} r_{11 ; \sigma^{\prime} \sigma} e^{-i k_{\sigma^{\prime}} x}\right]\left|\sigma^{\prime}\right\rangle
$$

on the left and

$$
\psi^{1 \sigma}(x)=\sum_{\sigma^{\prime}} \sqrt{\frac{k_{\sigma}}{k_{-\sigma^{\prime}}}} t_{12 ; \sigma^{\prime} \sigma} e^{i k_{-\sigma^{\prime}} x}\left|\sigma^{\prime}\right\rangle,
$$

on the right. The wave vectors are given by $k_{\sigma}$ $=\sqrt{2 m(\epsilon+\sigma \Delta / 2) / \hbar^{2}}$, with $\epsilon$ as the electron energy. (Note that the indices $\sigma, \sigma^{\prime} \in\{\uparrow, \downarrow\}$ refer to spin projections on the $z$ axis, and that the respective number $\sigma$ takes on $\{+1,-1\}$.)

We assume now that the amplitudes $r_{\sigma^{\prime} \sigma}$ and $t_{\sigma^{\prime} \sigma}$ for a domain wall with zero chirality are given, and we will determine them numerically later. (See Refs. 64 and 65 for analytical expressions for these coefficients, valid for large and small $k_{F} \lambda$, respectively.) All we need is that if we move the domain wall to position $r_{\mathrm{dw}}$, the wave function $\psi\left(x-r_{\mathrm{dw}}\right)$ is a solution of the Schrödinger equation for an electron moving in the displaced domain-wall texture. From this we deduce the transmission and reflection amplitudes for arbitrary domain-wall position. Furthermore, if the domain-wall precession angle $\phi_{0}$, i.e., its chirality, becomes nonzero, the magnetization texture changes to $\tilde{\mathbf{\Omega}}(x)$ $=\left[\sin \theta_{\mathrm{dw}}(x) \cos \phi_{0}, \sin \theta_{\mathrm{dw}}(x) \sin \phi_{0}, \cos \theta_{\mathrm{dw}}(x)\right]$. The solution of the Schrödinger equation for the wave function $\widetilde{\psi}(x)$ of electrons moving in this texture is given by

$$
\tilde{\psi}(x)=\left(\begin{array}{cc}
e^{-i \phi_{0} / 2} & 0 \\
0 & e^{i \phi_{0} / 2}
\end{array}\right) \psi(x),
$$

with $\psi(x)$ as the solution of the Schrodinger equation in Eq. (22) for a zero-chirality $\left(\phi_{0}=0\right)$ domain wall. This is sufficient to determine the chirality dependence of the transmission and reflection amplitudes.

Using these ingredients, we find for the reflection amplitude for electrons off a domain wall at arbitrary position and with arbitrary chirality that

$$
\mathbf{r}_{11}=\left(\begin{array}{ll}
r_{\uparrow \uparrow} e^{2 i k_{\uparrow} r_{\mathrm{dw}}} & r_{\uparrow \downarrow} e^{i\left(k_{\uparrow}+k_{\downarrow}\right) r_{\mathrm{dw}}-i \phi_{0}} \\
r_{\downarrow \uparrow} e^{i\left(k_{\uparrow}+k_{\downarrow}\right) r_{\mathrm{dw}}+i \phi_{0}} & r_{\downarrow \downarrow} e^{2 i k_{\downarrow} r_{\mathrm{dw}}}
\end{array}\right)
$$

and for the transmission amplitude

$$
\mathbf{t}_{21}=\left(\begin{array}{ll}
t_{\uparrow \uparrow} e^{i\left(k_{\uparrow}-k_{\downarrow}\right) r_{\mathrm{dw}}} & t_{\uparrow \downarrow} e^{-i \phi_{0}} \\
t_{\downarrow \uparrow} e^{i \phi_{0}} & t_{\downarrow \downarrow} e^{i\left(k_{\downarrow}-k_{\uparrow}\right) r_{\mathrm{dw}}}
\end{array}\right) .
$$

The reflection and transmission amplitudes for electrons coming in from the right reservoir, denoted by $\mathbf{r}_{22}$ and $\mathbf{t}_{12}$, respectively, are determined in the same way and are given by similar expressions. Because of the symmetries of the domain wall we further have that $r_{11 ; \sigma \sigma}=r_{22 ;-\sigma-\sigma}$, and $t_{21 ; \sigma \sigma}$ $=t_{12 ;-\sigma-\sigma}$. Furthermore, $r_{11 ; \sigma \sigma^{\prime}}=-r_{22 ; \sigma^{\prime} \sigma}$ and $t_{21 ; \sigma \sigma^{\prime}}=-t_{12 ; \sigma^{\prime} \sigma}$ for $\sigma \neq \sigma^{\prime}$. The final results are given by

$$
\mathbf{r}_{22}=\left(\begin{array}{ll}
r_{\downarrow \downarrow} e^{-2 i k_{\uparrow} r_{\mathrm{dw}}} & -r_{\downarrow \uparrow} e^{-i\left(k_{\uparrow}+k_{\downarrow}\right) r_{\mathrm{dw}}-i \phi_{0}} \\
-r_{\uparrow \downarrow} e^{-i\left(k_{\uparrow}+k_{\downarrow}\right) r_{\mathrm{dw}}+i \phi_{0}} & r_{\downarrow \downarrow} e^{-2 i k_{\downarrow} r_{\mathrm{dw}}}
\end{array}\right)
$$

and for the transmission amplitude

$$
\mathbf{t}_{12}=\left(\begin{array}{ll}
t_{\downarrow \downarrow} e^{i\left(k_{\uparrow}-k_{\downarrow}\right) r_{\mathrm{dw}}} & -t_{\downarrow \uparrow} e^{-i \phi_{0}} \\
-t_{\uparrow \downarrow} e^{i \phi_{0}} & t_{\uparrow \uparrow} e^{i\left(k_{\downarrow}-k_{\uparrow}\right) r_{\mathrm{dw}}}
\end{array}\right) .
$$

Insertion of the results for the transmission and reflection amplitudes into the expression for the current in Eq. (20), and identifying $X_{1}=r_{\mathrm{dw}}$ and $X_{2}=\phi_{0}$, yields the result

$$
\begin{aligned}
I_{1}= & -I_{2}=-\frac{|e|}{2 \pi}\left[\sum _ { \sigma } \left(2\left|r_{\sigma \sigma}\right|^{2}+\left|r_{\sigma-\sigma}\right|^{2}+\left|r_{-\sigma \sigma}\right|^{2}-\left|t_{\sigma \sigma}\right|^{2}\right.\right. \\
& \left.\left.+\left|t_{-\sigma-\sigma}\right|^{2}\right) k_{\sigma}\right] \frac{d r_{\mathrm{dw}}(t)}{d t}-\frac{|e|}{2 \pi}\left(\left|r_{\downarrow \uparrow}\right|^{2}-\left|r_{\uparrow \downarrow}\right|^{2}-\left|t_{\downarrow \uparrow}\right|^{2}\right. \\
& \left.+\left|t_{\uparrow \downarrow}\right|^{2}\right) \frac{d \phi_{0}(t)}{d t}
\end{aligned}
$$

containing again the two contributions, proportional to $d r_{\mathrm{dw}}(t) / d t$ and $d \phi_{0}(t) / d t$, respectively. The above derivation clearly shows that both these contributions are present in general.

Figure 3 gives the result for the voltage ${ }^{66}$ (that now includes the effect of the presence of the domain wall on the conductance)

$$
\Delta V \equiv \frac{-I_{1}}{\frac{2 \pi|e|^{2}}{\hbar}\left(\sum_{\sigma}\left|t_{\sigma \sigma}\right|^{2}+\left|t_{\sigma-\sigma}\right|^{2}\right)}
$$

for the various values of $k_{F} \lambda$ and for the exchange splitting $\Delta / \epsilon_{F}=0.5$ for the case of a field-driven domain wall which 


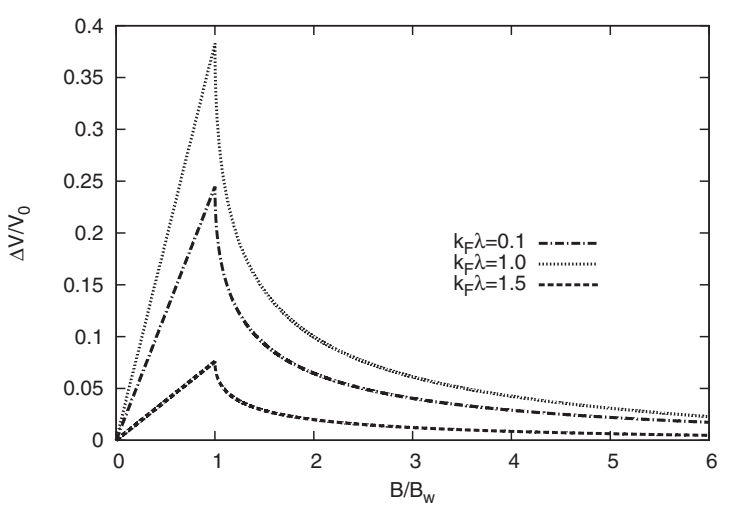

FIG. 3. Voltage induced by a moving domain wall for Gilbert damping $\alpha_{G}=0.01$, for $\Delta / \epsilon_{F}=0.5$, and for various values of $k_{F} \lambda$. The voltage is normalized to $V_{0}=g \mu_{B} B_{W} /|e|$. The magnetic field $B$ is in units of the Walker breakdown field $B_{W}$. For typical experiments we have that $V_{0} \sim 0.5 \mu \mathrm{V}$.

exhibits Walker breakdown. In this situation we have that ${ }^{44}$

$$
\begin{gathered}
\frac{d \phi_{0}(t)}{d t}=\frac{1}{\left(1+\alpha_{G}^{2}\right)} \frac{g \mu_{B} B_{W}}{\hbar} \operatorname{Re}\left[\sqrt{\left(\frac{B}{B_{W}}\right)^{2}-1}\right], \\
\frac{1}{\lambda} \frac{d r_{\mathrm{dw}}(t)}{d t}=\frac{g \mu_{B} B}{\alpha_{G} \hbar}-\frac{1}{\alpha_{G}} \frac{d \phi_{0}(t)}{d t} .
\end{gathered}
$$

The various transmission and reflection amplitudes are determined by numerically solving the Schrödinger equation in Eq. (22) for the specific domain-wall profile ${ }^{16}$

$$
\theta_{\mathrm{dw}}(x)=2 \tan ^{-1}\left(e^{x / \lambda}\right) .
$$

This texture corresponds to a domain wall that interpolates between a domain with magnetization pointing in the $+z$ direction and a domain with magnetization in the opposite direction (see Fig. 1).

The induced voltage in Fig. 3 is for fields below Walker breakdown qualitatively similar to that in Fig. 2 for the diffusive case. The voltage is largest when $k_{F} \lambda \simeq 1$. This is to be expected as the domain-wall resistance is also largest for that situation, ${ }^{64,65}$ and therefore nonadiabatic effects are also large. We also find that the voltage becomes larger upon increasing the ratio $\Delta / \epsilon_{F}$.

The ballistic model presented here underestimates the voltage for fields above Walker breakdown. This is most easily understood by taking the adiabatic limit $k_{F} \lambda \rightarrow \infty$. In that case all the reflection coefficients $r_{\sigma \sigma^{\prime}} \rightarrow 0$, the diagonal transmission coefficients $t_{\sigma \sigma} \rightarrow 0$, and the off-diagonal transmission coefficients $t_{\sigma \sigma^{\prime}} \rightarrow 1$. In this limit we find that, according to Eq. (30), the induced current and voltage become zero. This is a result of the simplicity of the model presented here and could be repaired by incorporating more transverse channels. (See Refs. 20 and 67 for a discussion of subtleties in describing spin-polarized transport with the LandauerBüttiker formalism.) To establish connection of the result of our simple ballistic model with the result found in the diffusive limit, it is easiest to put the spin polarization of the current in by hand. This is done by weighing the spin-up and down electrons coming from the left reservoir differently. In the adiabatic limit this amounts to taking $\left|t_{\downarrow \uparrow}\right|^{2}=(1+\mathcal{P}) / 2$, $\left|t_{\uparrow \downarrow}\right|^{2}=(1-\mathcal{P}) / 2$, and putting all other coefficients equal to zero. With this modification the result in Eqs. (30) and (31) reduces to that in Eq. (8) with $\beta_{\mathrm{sr}}=\beta_{\mathrm{na}}=0$.

We end by remarking that the ballistic model presented in this section applies when the phase-coherence length of the electrons is at least on the order of the domain-wall width. Also, because we have taken only one (spin-resolved) transport channel our calculation applies only to very tightly confined structures such as ferromagnetic nanocontacts. ${ }^{68}[\mathrm{Al}-$ though it may well be that the model for the domain-wall motion in Eq. (32) needs to be refined to apply to such situations.] Nonetheless, the method presented in this section should, when modified to apply to more realistic geometries, be a convenient starting point for addressing the ballistic case should experiments approach this limit in the near future.

\section{DISCUSSION AND CONCLUSIONS}

We have discussed the effects of nonadiabaticity on the voltage induced by a field-driven domain wall, and we considered both the diffusive and ballistic regimes of electronic transport. In particular, we have shown that incorporating effects of nonadiabaticity of the wall on the induced voltage is done by the same renormalization that incorporates nonadiabaticity in the description of current-induced propagation.

Future work will include incorporating also the effects of anisotropic magnetoresistance on the induced voltage and more sophisticated models of domain-wall motion, such as vortex walls. (We mention however that the rigid domainwall model discussed in this paper is known to give sensible results below Walker breakdown. ${ }^{69}$ ) For vortex walls the nonadiabatic effects mentioned in this paper likely play a less important role. Another interesting subject for study is the effect of spin relaxation and nonadiabaticity on the motive forces in mesoscopic rings, ${ }^{37}$ where these effects may actually be absent.

We hope that the present work, as well as previous theoretical work, ${ }^{14,37-39,42,46,47,49,60}$ will motivate further experiments ${ }^{50}$ on observing motive forces and voltages induced by time-dependent magnetization textures. One interesting aspect is the possibility of determining the degree of nonadiabaticity and effects of spin relaxation directly from such experiments. ${ }^{39}$

\section{ACKNOWLEDGMENTS}

It is a great pleasure to thank Stewart Barnes, Gerrit Bauer, Mathias Kläui, Hiroshi Kohno, Ties Lucassen, Allan MacDonald, Sadamichi Maekawa, Wayne Saslow, Junya Shibata, Mark Stiles, Henk Stoof, Henk Swagten, Gen Tatara, and Yaroslav Tserkovnyak for discussions. Furthermore, I am indebted to Qian Niu and Shengyuan Yuang for sharing their paper prior to publication. I would also like to gratefully acknowledge Henk Stoof for carefully proofreading the paper. This work was supported by the Nederlandse Organisatie voor Wetenschappelijke Onderzoek (NWO). 
*r.a.duine@uu.nl; http://www.phys.uu.nl/ duine

${ }^{1}$ J. C. Slonczewski, J. Magn. Magn. Mater. 159, L1 (1996).

${ }^{2}$ L. Berger, Phys. Rev. B 54, 9353 (1996).

${ }^{3}$ M. Tsoi, A. G. M. Jansen, J. Bass, W.-C. Chiang, M. Seck, V. Tsoi, and P. Wyder, Phys. Rev. Lett. 80, 4281 (1998).

${ }^{4}$ E. B. Myers, D. C. Ralph, J. A. Katine, R. N. Louie, and R. A. Buhrman, Science 285, 867 (1999).

${ }^{5}$ Ya. B. Bazaliy, B. A. Jones, and Shou-Cheng Zhang, Phys. Rev. B 57, R3213 (1998).

${ }^{6}$ J. Fernández-Rossier, M. Braun, A. S. Nuńẽz, and A. H. MacDonald, Phys. Rev. B 69, 174412 (2004).

${ }^{7}$ D. C. Ralph and M. D. Stiles, J. Magn. Magn. Mater. 320, 1190 (2008).

${ }^{8}$ S. Zhang and Z. Li, Phys. Rev. Lett. 93, 127204 (2004).

${ }^{9}$ S. E. Barnes and S. Maekawa, Phys. Rev. Lett. 95, 107204 (2005); S. E. Barnes, ibid. 96, 189701 (2006).

${ }^{10}$ Y. Tserkovnyak, H. J. Skadsem, A. Brataas, and G. E. W. Bauer, Phys. Rev. B 74, 144405 (2006).

${ }^{11}$ H. Kohno, G. Tatara, and J. Shibata, J. Phys. Soc. Jpn. 75, 113706 (2006).

${ }^{12}$ F. Piéchon and A. Thiaville, Phys. Rev. B 75, 174414 (2007).

${ }^{13}$ R. A. Duine, A. S. Núñez, Jairo Sinova, and A. H. MacDonald, Phys. Rev. B 75, 214420 (2007).

${ }^{14}$ Y. Tserkovnyak, A. Brataas, and G. E. W. Bauer, J. Magn. Magn. Mater. 320, 1282 (2008).

${ }^{15}$ Yaroslav Tserkovnyak and Clement H. Wong, arXiv:0806.4656 (unpublished).

${ }^{16}$ G. Tatara and H. Kohno, Phys. Rev. Lett. 92, 086601 (2004); 96, 189702 (2006).

${ }^{17}$ G. Tatara, H. Kohno, J. Shibata, Y. Lemaho, and K.-J. Lee, J. Phys. Soc. Jpn. 76, 054707 (2007).

${ }^{18}$ G. Tatara, H. Kohno, and J. Shibata, Phys. Rep. 468, 213 (2008).

${ }^{19}$ Here, nonadiabatic refers to terms beyond linear response in spatial gradients of the magnetization direction, considering, however, only first order in temporal derivatives.

${ }^{20}$ X. Waintal and M. Viret, Europhys. Lett. 65, 427 (2004).

${ }^{21}$ M. Thorwart and R. Egger, Phys. Rev. B 76, 214418 (2007).

${ }^{22}$ A. K. Nguyen, H. J. Skadsem, and A. Brataas, Phys. Rev. Lett. 98, 146602 (2007).

${ }^{23}$ J. Grollier, P. Boulenc, V. Cros, A. Hamzi, A. Vaurès, A. Fert, and G. Faini, Appl. Phys. Lett. 83, 509 (2003).

${ }^{24}$ M. Tsoi, R. E. Fontana, and S. S. P. Parkin, Appl. Phys. Lett. 83, 2617 (2003).

${ }^{25}$ A. Yamaguchi, T. Ono, S. Nasu, K. Miyake, K. Mibu, and T. Shinjo, Phys. Rev. Lett. 92, 077205 (2004).

${ }^{26}$ M. Kläui, C. A. F. Vaz, J. A. C. Bland, W. Wernsdorfer, G. Faini, E. Cambril, L. J. Heyderman, F. Nolting, and U. Rüdiger, Phys. Rev. Lett. 94, 106601 (2005).

${ }^{27}$ G. S. D. Beach, C. Knutson, C. Nistor, M. Tsoi, and J. L. Erskine, Phys. Rev. Lett. 97, 057203 (2006).

${ }^{28}$ M. Hayashi, L. Thomas, C. Rettner, R. Moriya, and S. S. P. Parkin, Nat. Phys. 3, 21 (2007).

${ }^{29}$ M. Yamanouchi, D. Chiba, F. Matsukura, and H. Ohno, Nature (London) 428, 539 (2004).

${ }^{30}$ M. Yamanouchi, D. Chiba, F. Matsukura, T. Dietl, and H. Ohno, Phys. Rev. Lett. 96, 096601 (2006).

${ }^{31}$ A. Thiaville, Y. Nakatani, J. Miltat, and Y. Suzuki, Europhys. Lett. 69, 990 (2005).
${ }^{32}$ A. Rebei, W. N. G. Hitchon, and G. J. Parker, Phys. Rev. B 72, 064408 (2005).

${ }^{33}$ J.-I. Ohe and B. Kramer, Phys. Rev. Lett. 96, 027204 (2006).

${ }^{34}$ Jiang Xiao, A. Zangwill, and M. D. Stiles, Phys. Rev. B 73, 054428 (2006).

${ }^{35}$ R. A. Duine, A. S. Núñez, and A. H. MacDonald, Phys. Rev. Lett. 98, 056605 (2007).

${ }^{36}$ L. Heyne, M. Kläui, D. Backes, T. A. Moore, S. Krzyk, U. Rüdiger, L. J. Heyderman, A. F. Rodríguez, F. Nolting, T. O. Mentes, M. Á. Niño, A. Locatelli, K. Kirsch, and R. Mattheis, Phys. Rev. Lett. 100, 066603 (2008).

${ }^{37}$ A. Stern, Phys. Rev. Lett. 68, 1022 (1992).

${ }^{38}$ S. E. Barnes and S. Maekawa, Phys. Rev. Lett. 98, 246601 (2007).

${ }^{39}$ R. A. Duine, Phys. Rev. B 77, 014409 (2008).

${ }^{40}$ Yaroslav Tserkovnyak and Matthew Mecklenburg, Phys. Rev. B 77, 134407 (2008).

${ }^{41}$ A. Auerbach, Interacting Electrons and Quantum Magnetism (Springer-Verlag, New York, 1994).

${ }^{42}$ W. M. Saslow, Phys. Rev. B 76, 184434 (2007).

${ }^{43}$ Note that there is a sign error in Eq. (15) of Ref. 39.

${ }^{44}$ N. L. Schryer and L. R. Walker, J. Appl. Phys. 45, 5406 (1974).

${ }^{45}$ G. S. D. Beach, C. Nistor, C. Knutson, M. Tsoi, and J. L. Erskine, Nature Mater. 4, 741 (2005).

${ }^{46}$ L. Berger, Phys. Rev. B 33, 1572 (1986).

${ }^{47}$ Jun-ichiro Ohe, A. Takeuchi, and G. Tatara, Phys. Rev. Lett. 99, 266603 (2007).

${ }^{48}$ Shengyuan A. Yang, Di Xiao, and Qian Niu, arXiv:0709.1117 (unpublished).

${ }^{49}$ M. Stamenova, T. N. Todorov, and S. Sanvito, Phys. Rev. B 77, 054439 (2008).

${ }^{50}$ S. A. Yuang, G. S. D. Beach, C. Knutson, D. Xiao, Q. Niu, M. Tsoi, and J. L. Erskine (unpublished).

${ }^{51}$ M. Büttiker, Y. Imry, R. Landauer, and S. Pinhas, Phys. Rev. B 31, 6207 (1985).

${ }^{52}$ C. Bruder and H. Schoeller, Phys. Rev. Lett. 72, 1076 (1994).

${ }^{53}$ P. W. Brouwer, Phys. Rev. B 58, R10135 (1998).

${ }^{54}$ M. Switkes, C. M. Marcus, K. Campman, and A. C. Gossard, Science 283, 1905 (1999).

${ }^{55}$ Susan K. Watson, R. M. Potok, C. M. Marcus, and V. Umansky, Phys. Rev. Lett. 91, 258301 (2003).

${ }^{56}$ Y. Tserkovnyak, A. Brataas, and G. E. W. Bauer, Phys. Rev. Lett. 88, 117601 (2002).

${ }^{57}$ Y. Tserkovnyak, A. Brataas, and G. E. W. Bauer, Phys. Rev. B 66, 224403 (2002).

${ }^{58}$ S. Mizukami, Y. Ando, and T. Miyazaki, J. Magn. Magn. Mater. 226-230, 1640 (2001).

${ }^{59}$ M. V. Costache, M. Sladkov, S. M. Watts, C. H. van der Wal, and B. J. van Wees, Phys. Rev. Lett. 97, 216603 (2006).

${ }^{60}$ Jiang Xiao, Gerrit E. W. Bauer, and Arne Brataas, Phys. Rev. B 77, 180407(R) (2008).

${ }^{61}$ M. Büttiker, H. Thomas, and A. Prêtre, Z. Phys. B: Condens. Matter 94, 133 (1994).

${ }^{62}$ Note that $I_{\nu}$ is a current with units $\left[I_{\nu}\right]=\mathrm{C} / \mathrm{s}$, whereas $j$ is strictly speaking a current density with $[j]=\mathrm{C} / \mathrm{m}^{2} \mathrm{~s}$.

${ }^{63}$ J. B. A. N. van Hoof, K. M. Schep, A. Brataas, G. E. W. Bauer, and P. J. Kelly, Phys. Rev. B 59, 138 (1999).

${ }^{64}$ G. Tatara, J. Phys. Soc. Jpn. 69, 2969 (2000).

${ }^{65}$ V. K. Dugaev, J. Berakdar, and J. Barnas, Phys. Rev. B 68, 104434 (2003). 
${ }^{66}$ See S. M. L. Polianski and P. W. Brouwer, Phys. Rev. B 64, 075304 (2001) for a discussion on the relation between voltage and current for a quantum-dot pump.

${ }^{67}$ I. I. Mazin, Phys. Rev. Lett. 83, 1427 (1999).
${ }^{68}$ N. García, M. Muñoz, and Y.-W. Zhao, Phys. Rev. Lett. 82, 2923 (1999); P. Bruno, ibid. 83, 2425 (1999).

${ }^{69}$ G. S. D. Beach, M. Tsoi, and J. L. Erskine, J. Magn. Magn. Mater. 320, 1272 (2008). 